\title{
EDUCACIÓN PARA LA CIUDADANÍA Y LA CONVIVENCIA
}

\author{
Ascensión Palomares Ruiz \\ Universidad de Castilla-La Mancha
}

\section{RESUMEN}

La educación puede considerarse básicamente como consolidación de valores esenciales para la vida de la persona en la sociedad en que ha de integrarse. Su papel adquiere una gran trascendencia en esta época de crisis, en la que ciertos valores genéricos e, incluso, avances tecnológicos están imperando sobre lo específicamente humano, por lo que hay que desarrollar actitudes que permitan configurar una nueva cultura, que nos libre de la deshumanización y potencie la lucha por lo colectivo, favoreciendo la prelación de los intereses generales sobre los individuales o sectoriales.

Todo docente ha de saber que su tarea trasciende la simple reproducción de determinados contenidos curriculares, para alcanzar conceptos significativos, despertando en el alumnado actitudes de participación en la configuración de la sociedad en que está inmerso.

Nos encontramos ante un problema social que requiere un planteamiento holístico, en el que los primeros en cambiar actitudes deben ser nuestros representantes públicos, sindicatos incluidos. No debemos olvidar que la mayoría de los alumnos que muestran conductas agresivas en la infancia suelen convertirse en adultos violentos. Por tanto, la educación para la paz, la tolerancia y la solidaridad, desde la más temprana edad, constituyen el mejor medio para prevenir estas situaciones y la también preocupante violencia doméstica. Una tarea en la que deben trabajar colaborativamente, no sólo los padres y docentes, sino la sociedad, en general.

Palabras clave: Tolerancia, inmigración, violencia, educación para la paz, valores, acoso escolar, solidaridad, interculturalidad, exclusión social.

\section{ABSTRACT}

Education can be basically considered as the consolidation of a set of values which are essential in the life of a person right inside the society where they have to get integrated. It acquires a great transcendence in this crisis times, in which certain generic values and even technological advance are imposing themselves 
over what is specifically human, so it is necessary to develop new attitudes which lead to a new kind of culture, which sets us free from dehumanization and encourages the fight for the collective interests, and favours them over the individual or partial ones.

In the field of education, every professional must be aware of the fact that their task transcends the mere communication of some curricular contents and must reach meaningful concepts and aim to the awakening of participative behaviour in the construction of the surrounding society.

We are facing a social problem which requires a holistic treatment, in which our public representatives -and this include trades- should change their attitudes first. We should not forget the fact that most pupils showing aggressive behaviour become violent adults. Therefore, education for peace, tolerance and solidarity from the youngest age, are the best way to prevent these situations and also the worrying domestic violence. This is a task in which everyone, not only parents and teachers, but the whole society, should work in cooperation.

Key words: Tolerance, immigration, violence, education for peace, values, bullying, solidarity, intercultural issues, social exclusion.

\section{La violencia escolar, un grave problema social}

La violencia en la Escuela no es un problema nuevo; pero, tras el triste final de un alumno de 14 años de Hondarribia (Guipúzcoa), que sufrió maltrato psicológico y físico de sus compañeros de Instituto, se dispararon las alarmas sociales, políticas y educativas, generando múltiples debates, seminarios, estudios, etc. Sin embargo, no sólo no se ha conseguido frenar el problema, sino que los casos de acoso escolar afloran como algo natural y normal, en una sociedad cada vez más competitiva e insolidaria.

En la actualidad, nuestras autoridades públicas, tanto del poder ejecutivo como del legislativo y judicial, son las primeras en ofrecernos claros ejemplos de violencia verbal y acoso psicológico contra el adversario político o cualquier persona digna que defiende la equidad y la justicia, disintiendo del comportamiento sectario o partidista de quienes ostentan el poder. En este espectacular contexto de virulencia verbal, las descalificaciones personales y las técnicas de "mobbing" político y mediático que se nos ofrecen -día a día- en los medios de comunicación posiblemente incidan en un incremento de la violencia, en los ámbitos escolar, familiar, laboral, etc.

Diariamente, se difunden informaciones sobre nuevas víctimas de la violencia en los Centros escolares, tanto públicos como privados, generando una alarma social que precisa un amplio análisis -con rigor científico y metodológico- de los datos ofrecidos.

En los Centros escolares, el término inglés "bullying" se refiere a una serie de actos -intencionados y con una cierta continuidad- que desarrollan unos alumnos contra otros. Al igual que el "mobbing", se inicia de una manera sutil, casi imperceptible, que - poco a poco- se va agravando, conllevando -a veces- maltrato físico. Se suele comenzar con los apodos, risas ante equivocaciones, burlas sobre la apariencia física, acusación de hechos no realizados, ignorancia de presencia evidente, etc., que son las for- 
mas más usuales de hostigar a un/a compañero/a de colegio. Los principales indicadores que nos facilitan información, en caso de "bullying", son: agresiones, desprecio-ridiculización, intimidación-amenazas, exclusión-bloqueo social, hostigamiento verbal, coacción y robos.

Conviene resaltar que el acoso psicológico -sin huellas físicas externas- es más peligroso y difícil de detectar. Además, puede generar un cuadro clínico, con una sintomatología que requiere terapia.

Los alumnos y alumnas son acosados prácticamente por igual; pero existen diferencias en la forma en que se produce el maltrato: ellas suelen recibir mayor maltrato psicológico, mientras que, en ellos, predomina el acoso físico.

El alumnado que sufre "bullying" no siempre dispone de un contexto familiar o escolar que le posibilite informar -desde el principio- del posible acoso que está padeciendo. Suelen ser personas introvertidas, que sienten vergüenza de su propio comportamiento, al no tener la "valentía" de enfrentarse a sus compañeros. Además, buscan excusas para no ir a la Escuela, vuelven a su casa Ilorando, tienden al aislamiento e, incluso, padecen trastornos psicofísicos.

Los datos -como siempre- varían en función de la fuente de procedencia y del enfoque utilizado a la hora de analizar el problema. Sin embargo, hay unos hechos evidentes: siempre ha existido el acoso escolar, cada día se va extendiendo más y no se están implementando las medidas preventivas, correctivas y terapéuticas adecuadas. Sin ánimo de exhaustividad, cabe destacar algunos de los estudios más significativos existentes: una encuesta del Instituto de la Juventud (INJUVE) eleva el porcentaje de víctimas de violencia física y/o psicológica habitual a un 3\% del alumnado, mientras un $16 \%$ de los encuestados reconoce haber participado en exclusiones o en agresiones psicológicas a otros compañeros.

No debemos olvidar que hace cinco años, en el informe presentado por el Defensor del Pueblo sobre "Violencia escolar, el maltrato entre iguales", ya se indicaba que un 33'8\% del alumnado de Educación Secundaria había padecido, en algún momento, acoso por parte de sus compañeros. De ellos, al menos, el 4'5\% reconocía haber sufrido agresión física. En un informe posterior del Instituto de Evaluación y Asesoramiento Educativo (IDEA), se reflejaba que el $49 \%$ de los alumnos afirmaba haber sido insultado o criticado, aunque sólo el 12\% manifestaba que había sufrido agresión física y un 13\% confesaba haber pegado a sus compañeros.

En las conclusiones del estudio "Cisneros" (septiembre de 2005), sobre violencia y acoso escolar en la Comunidad de Madrid, se asegura que, entre 7 y 18 años, uno de cada cuatro escolares son acosados por sus compañeros y los alumnos de Educación Primaria padecen un acoso siete veces superior al resto de los escolares.

Resulta curioso -como en otros relacionados con la violencia familiar o laboral- esa especie de pacto de silencio de las personas que suelen -y pueden- conocer la situación de acoso. Los estudios realizados permiten comprobar que la dinámica del "bullying" se ve favorecida por la pasividad de otros compañeros, que -a pesar de la evidencia del problema- se convierten en observadores interesados e inhibidos. Tal actitud tiene un componente defensivo, a fin de intentar evitar ser otro posible blanco de los 
ataques, convirtiéndose así en cómplices pasivos de la situación. Al no apoyar a la víctima de acoso, en cierta medida, sus compañeros están generando y manteniendo las situaciones violentas. Evidentemente, la violencia en las aulas no es una conducta desconocida, sino oculta y silenciada, aunque pase inadvertida para los adultos. En un estudio realizado por investigadores de la Universidad Complutense de Madrid (2007), indican que, un 48\% manifiesta que intervendría, aunque la víctima no fuese amigo suyo. Un porcentaje superior al indicado en el estudio de Defensor del Pueblo, con datos de 1999 a 2006, que lo situaba en un 30\%.

En este sentido, los resultados de las investigaciones que he realizado no ofrecen una visión de descenso porcentual, sino de ligero aumento, situándose -en CLM, actualmente- el porcentaje de alumnos adolescentes agresores en un 5'5\%. Es importante destacar que, en todos los estudios publicados, los resultados son similares, resaltando el alto número de adolescentes que se manifiesta en contra de la violencia (76\%); pero sólo un 36\% indica que actuaría ante un problema de acoso. En el Cuadro 1, se resumen las conclusiones de la investigación realizada en CLM, durante el curso 2006-7.

\begin{tabular}{|c|c|c|}
\hline $\begin{array}{l}\text { CONTRA LA } \\
\text { VIOLENCIA } \\
(76 \%)\end{array}$ & $\begin{array}{l}\text { INDIFERENTE } \\
\left(18^{\prime} 5 \%\right)\end{array}$ & $\begin{array}{c}\text { PARTICIPA EN LA } \\
\text { VIOLENCIA } \\
\left(5^{\prime} 5 \%\right)\end{array}$ \\
\hline $\begin{array}{c}\text { RECHÄZA Y ACTÚA EN } \\
\text { LOS CASOS QUE } \\
\text { CONOCE } \\
36 \%\end{array}$ & $\begin{array}{l}\text { NO ES SU PROBLEMA } \\
\qquad 11^{\prime} 5 \%\end{array}$ & $\begin{array}{c}\text { DIRIGE EL GRUPO } \\
\text { VIOLENTO } \\
0 ' 8 \%\end{array}$ \\
\hline $\begin{array}{c}\text { CONOCE EL PROBLEMA, } \\
\text { LO RECHAZA, PERO NO } \\
\text { ACTUA } \\
40 \%\end{array}$ & $\begin{array}{c}\text { NO CONOCE NINGÛN } \\
\text { CASO } \\
7 \%\end{array}$ & $\begin{array}{c}\text { PERTENECE AL GRUPO } \\
47 \%\end{array}$ \\
\hline
\end{tabular}

Cuadro 1

En todos los países de nuestro entorno, desde hace años, se vienen experimentando diversas medidas para paliar la violencia en los Centros de enseñanza. A título indicativo, los maestros noruegos deben suscribir un "manifiesto contra el acoso", comprometiéndose a trabajar para impedir -en la media de sus posibilidades- el "bullying", por lo que asumen las responsabilidades de cualquier tipo de acoso que pueda producirse en sus aulas. Algunos especialistas, como el profesor Dan Olweus, fundador del Área de 
Investigación sobre acoso escolar en la Universidad de Bergen (Noruega), indican que el "bullying" es responsabilidad principal de la escolaridad, por lo que debe solucionarse desde el mismo Centro educativo, destacando el papel clave que ejercen los profesores y el equipo directivo, para aumentar el conocimiento del personal de la escuela sobre este problema, transmitir medidas de trabajo, imponer disciplina en las aulas, etc. En definitiva, cambiar el clima escolar.

En nuestro país, a pesar de la evidencia del problema, mostrado en diversas estadísticas, la realidad es que -hasta ahora- se ha hecho muy poco para afrontar con efectividad este tipo de violencia, en los diferentes ámbitos. Como ejemplos más significativos, habría que destacar la iniciativa destinada a prevenir el acoso escolar - "Buen rollo"del Departamento de Educación de la Generalitat de Cataluña, dirigida al alumnado de Educación Primaria y Secundaria. Otras, como la llevada a cabo por la Cruz Roja Juventud de Pontevedra, bajo el lema "Sin violencia, convivencia", que organiza cursos sobre acoso escolar, para profesores de Educación Secundaria.

Sin embargo, poco a poco, con la ayuda de diversas publicaciones, páginas Web, teléfonos, etc. la "cultura del silencio" se está superando y, aunque todavía resulta más fácil detectar a los abusadores que a las víctimas, éstas van cambiando y poseen más medios para poder superar el acoso que están sufriendo. En un Seminario sobre violencia en las aulas, realizado el 7 de octubre de 2005, en Valencia, se defendió la combinación de leyes y programas de prevención e intervención, para recuperar la autoridad del profesorado y acabar con la violencia escolar y el acoso entre el alumnado.

El Consejero de Educación de la Generalitat Valenciana reivindicó una iniciativa nacional -pilotada por el Gobierno central- para que todas las experiencias en materia de lucha contra la violencia y el acoso escolar fomentaran una cultura de convivencia en los Centros, con elementos comunes. En este sentido, se ha presentado el "PLAN PREVI", para la prevención de la violencia, iniciativa que pretende luchar contra esta problemática, con medidas de refuerzo del papel de los psicopedagogos, potenciación de las tutorías y mediación del conflicto, como elemento transversal en el que todos los miembros de la Escuela deben estar formados.

A través de una iniciativa promovida por "Protégeles" y el Defensor del Pueblo de la Comunidad de Madrid, a partir del día 20 de octubre de 2005, varios psicólogos están pendientes de las denuncias que hagan los escolares que se sientan sometidos a acoso, a través de correo electrónico. En dicha Web, hay una parte con información para padres y madres -y otra para el alumnado- sobre el acoso escolar. Los responsables de esta iniciativa apuestan porque los especialistas, a partir de los mensajes enviados por el alumnado, deriven los casos más graves de agresiones al Defensor del Menor o a las autoridades pertinentes y actúen, en el Centro educativo, cuando el alumnado facilite datos.

Hace un par de años, valorábamos positivamente la posible iniciativa del Ministerio de Educación, tendente a la incorporación a los Centros de la figura del Educador Social, para intervenir activamente y prevenir situaciones de riesgo y violencia producidas en los mismos. Dicha medida, difundida un tanto confusamente y sin la necesaria reglamentación, resultaba totalmente insuficiente, pero lo más lamentable es que prácticamente no se ha implantado. Además, se precisa formar adecuadamente al profesorado y disponer 
de otras iniciativas complementarias, que contribuyan a disminuir la creciente evolución violenta que se respira en la sociedad actual, empezando por la propia familia.

Por otra parte, un sistema más represivo, como el establecido en Francia, creó la figura del policía-tutor, con la función de trabajar con la dirección de los Centros y ayudar al alumnado que lo precise. Este modelo tiene grandes semejanzas a las propuestas que realizaban -hace años- algunos portavoces del Gobierno español, que parecían orientarse hacia una línea más tendente a lo represivo que a lo educativo, pues anunciaron cambios en la Ley del Menor, para endurecer los castigos por delitos graves y combatir fenómenos como las bandas juveniles. La reforma -según informaciones de los Ministerios de Justicia y de Interior- contemplaría medidas de alejamiento para el agresor, en casos de acoso escolar, y aumentaría los supuestos de internamiento -en régimen cerrado- a delitos graves, aunque no sean violentos, como el tráfico de drogas. Las informaciones facilitadas por los portavoces del Gobierno tienden a unir situaciones tan diferentes como el acoso escolar y el tráfico de drogas, en los Centros educativos, centrándose en sistemas represivos, para ambos casos, a pesar de ser dos fenómenos cuyos orígenes, tratamiento y corrección son muy diferentes, aunque -en algún momento- tengan elementos comunes. Es decir, desde las Administraciones Públicas, se continúa en una línea de inhibición, como en otros temas, sin afrontar seriamente el problema existente.

Además, en la UE, se está produciendo una forma específica de violencia con problemas de xenofobia, sobre todo contra africanos y asiáticos, así como aumento de bandas juveniles organizadas, similar a las conocidas en España. Por ello, sería conveniente emplazar - de nuevo- a las Administraciones públicas, para que Ileven a cabo un programa sobre el particular.

Resulta importante destacar que fruto de una decisión -aprobada por unanimidaden las Cortes de Castilla-La Mancha, a primeros de septiembre de 2006, se firmó el primer Acuerdo por la Convivencia Escolar, una iniciativa que ha contado con el respaldo de los agentes sociales, educativos y económicos de la región. La creación de un Observatorio Regional o la elaboración de la Carta de Convivencia son algunas de las iniciativas que contempla un acuerdo que supone un marco de actuación para implicar a toda la sociedad castellano-manchega en la prevención y lucha contra la violencia en las aulas y el fomento de las buenas relaciones.

Además, el acuerdo prevé otras medidas, entre ellas, la elección por parte del Consejo Escolar de cada Centro de un miembro adulto que ejerza las funciones de mediación y arbitraje en los procesos de conflictividad que se puedan generar. También está prevista la elaboración de Planes de Convivencia en los Centros, con la participación de todos los sectores educativos. Medidas en materia de formación, mediación y prevención, así como programas formativos en los medios de comunicación completan las previsiones del acuerdo, en cuya aplicación tendrán que trabajar junto las familias y los Centros, según el compromiso adquirido.

Hay que reconocer que se ha diseñado un marco adecuado para abordar eficazmente el problema de la violencia en las aulas. Esperemos que exista voluntad política clara y se disponga de los recursos humanos y materiales precisos para que constituya una realidad. 
Resulta urgente poner en funcionamiento en todos los Centros Educativos - desde el inicio de la escolaridad- las acciones precisas para prevenir el acoso, implementando programas educativos diseñados para fomentar un clima escolar de no violencia, tolerancia, respeto y solidaridad, y posibilitando al alumnado la adquisición de estrategias de resolución pacífica de conflictos, respeto a las diferentas individuales, y aceptación de la integración, sin ningún tipo de discriminación. Por ello, dentro de las actividades cotidianas resulta fundamental el aprendizaje cooperativo, evitando la competitividad y la violencia, desde la más temprana edad.

\section{Diseño de la investigación}

El aumento de la violencia en la sociedad es un grave problema que requiere una respuesta inmediata y un compromiso de todos los representantes de los diferentes sectores de la comunidad (Administraciones, medios de comunicación, sindicatos, profesorado, padres y madres, empresarios, ONGs, etc.). Por ello, en el año 2006, iniciamos una investigación con el objetivo de conocer la situación real en nuestra región y ofrecer instrumentos para que los docentes dispongan de medidas de prevención efectivas y facilitar una intervención precoz ante los problemas de violencia que se puedan presentar en los Centros escolares.

En los trabajos realizados, se han elaborado y aplicado diversos Cuestionarios (ANEXO I), entre los que habría que resaltar el relativo a la Evaluación de violencia escolar y bullying, diseñado para ser utilizado tanto por el profesorado como por padres y madres; y el de Violencia y educación en la sociedad actual, para alumnado de Educación Secundaria y Universidad. En dichos cuestionarios, se plantean una serie de cuestiones que deben responder, siendo la escala de valoración utilizada la siguiente: 1= nada; 2 = poco; 3 = bastante, $4=$ mucho.

El principal objetivo de la investigación se ha centrado como apoyo y potenciación de la docencia. En este sentido, se ha pretendido mejorar la tarea docente en la línea indicada por la UNESCO (1999):

"La relación entre la investigación científica, la educación, la innovación tecnológica y los beneficios prácticos es hoy mucho más diversificada y compleja, y a menudo intervienen en ella numerosos actores además de los investigadores. Ya no se puede justificar el progreso de la ciencia exclusivamente por la búsqueda de conocimientos. Debe también ser defendido con el argumento de su pertinencia y eficacia para responder a las necesidades y expectativas de nuestras sociedades".

La investigación se desarrolló durante el curso 2006-7, con alumnado de la Universidad de Castilla-La Mancha que cursa diversas carreras en el campus de Albacete. En curso 2008-09, se ha ampliado la muestra superando el 10\% de la población.

Los objetivos planteados son:

- Identificar los elementos clave para evitar la violencia en los Centros.

- Potenciar la autonomía del alumnado y las actitudes colaborativas.

- Formular propuestas concretas para que el alumnado sea responsable de su propio aprendizaje, a fin propiciar una convivencia pacifica y solidaria. 
- Analizar las competencias que se ponen en juego para conseguir que el alumnado de la Universidad interactúe social y profesionalmente con su entorno.

- Conocer las actividades que son más aceptadas por el alumnado, para el intercambio de experiencias y la comunicación con sus compañeros/as y con el profesorado.

El modelo de investigación más adecuado para la consecución de los objetivos planteados es de carácter no experimental propiamente dicho (Campbell y Stanley, 1978), estando principalmente basado en la aplicación de cuestionarios. Con este instrumento, se ha procurado obtener -de forma sistemática y ordenada-información sobre las variables que intervienen en la investigación de la realidad en que nos encontramos y -al tiempo- provocar la reflexión sobre su propia actitud personal y profesional. Consecuentemente, se ha comprobado que la fiabilidad del instrumento es muy alta.

De acuerdo con lo indicado por Guba (1989), se puede decir que la dimensión cualitativa de la investigación reúne los cuatro criterios de rigor relacionados con la credibilidad de la investigación: valor de verdad (credibilidad), aplicabilidad (transferibilidad), consistencia (dependencia) y neutralidad (confirmabilidad). Además, el rigor de la investigación está también garantizado, en lo que afecta a la dimensión cuantitativa de la investigación, la construcción de instrumentos, el control de todo el proceso y el rigor en la recogida de la información.

La población objeto de estudio queda definida por el conjunto de alumnos/as que cursan estudios en la Universidad de Castilla-La Mancha (UCLM) en la provincia de Albacete. La muestra -seleccionada al azar- está integrada por 202 alumnos/as (150 de Magisterio y 52 de otras carreras). La muestra resulta adecuada para este tipo de estudios; sin embargo, se está previsto ampliar la investigación a otras provincias de la Universidad de Castilla-la Mancha (Ciudad Real, Cuenca y Toledo), y diseñar otros Cuestionarios para poder recoger la opinión del profesorado y del alumnado que de Educación Primaria y de Educación Secundaria, a fin de profundizar en la investigación, contrastar los resultados y enriquecer -en la medida de lo posible- las propuestas de mejora.

Los cuestionarios utilizados se han pasado a las personas implicadas en el estudio, con el fin de recopilar informaciones de carácter general y han servido de base para la elaboración posterior de entrevistas. Además, dichas entrevistas personales han facilitado el conocimiento de determinados aspectos no recogidos en los cuestionarios.

En la elaboración de los cuestionarios ha tenido en cuenta, entre otros, los siguientes aspectos:

- Concreción de las variables del estudio, a partir de los objetivos planteados.

- Establecimiento de criterios e indicadores.

- Elaboración de cuestionarios abiertos y facilitadores de la reflexión crítica y la aportación del investigador y de los participantes en la misma.

- Validación de los cuestionarios y su elaboración definitiva.

La información obtenida se ha analizado en dos fases y con dos técnicas diferentes, siendo la primera de ellas de tipo cuantitativo y, la segunda, preferentemente cualitativa. El análisis cuantitativo ha tenido menor importancia, aunque ha posibilitado conocer:

- Deficiencias formativas. 
- Los intereses del alumnado y del profesorado respecto a la mejora de la convivencia pacífica en los Centros educativos y en la sociedad, en general.

- Las diferencias y similitudes entre el alumnado de las distintas carreras universitarias respecto a la experiencia y valoración sobre la convivencia en los Centros.

- La elaboración de propuestas de mejora, etc.

Los análisis descriptivos se han realizado mediante el programa SPSS, que ha facilitado -en algunos casos- información muy concreta sobre los indicadores establecidos y el grado de cumplimiento de cada uno de ellos.

Resulta evidente que todos los estudios e investigaciones realizadas recogen un aumento progresivo de casos de maltrato entre iguales (bullying) y que cada vez suelen ser más pequeños los alumnos acosados. Lo que parece claro es que, al menos, un $2{ }^{\prime} 5 \%$ del alumnado sufre acoso escolar de forma constante y que un $6^{\prime} 2 \%$ lo padece esporádicamente.

Por lo general, las Administraciones públicas tienden a minimizar los hechos, a buscar explicaciones y a sancionar -a la baja- al acosador, cuando no a inculpar al acosado (si se defiende), a asignarle un diagnóstico psicopatológico e -incluso- a que sea tratado farmacológicamente; es decir, se libera al acosador de su carga y se abandona al acosado a su suerte. Evidentemente, la violencia en las aulas no es una conducta desconocida, sino oculta y silenciada, aunque pase inadvertida para los adultos. En este sentido, se les preguntaba si habían actuado cuando se ha detectado algún caso de acoso. Solamente un 6\%, manifiesta haber actuado adecuadamente (Gráfico 4). Además, un 91\% manifiesta que se tiene miedo a denunciar a los acosadores.

Aparte de unas cuestiones previas para enmarcar el problema de la violencia en los Centros escolares y los lugares de acoso, así como la influencia de los medios de comunicación (Gráficos 1, 2 y 3), también se subraya la influencia del comportamiento de los responsables políticos en el aumento de la violencia, superando el 83\% (Gráfico 5).

Las Administraciones educativas no sólo no actúan con eficacia en los casos denunciados (Gráfico 6), sino que tampoco realizan un auténtico proceso de PREVENCIÓN del acoso en los Centros educativos (Gráfico 7).

La influencia de los videojuegos y la TV en el aumento de la violencia, queda evidente, dado que, el $89 \%$ valora que influye bastante o mucho (Gráfico 8). Además, el $61 \%$ indica que le gustan las películas con escenas violentas (Gráfico 14).

Igualmente, se preguntaba sobre la posible influencia de la violencia doméstica con el acoso en las aulas, concluyendo más del 96\% que existe una clara relación (Gráfico 9). Además, se insistía sobre la posible influencia de la situación familiar en el comportamiento violento, indicado un $89 \%$ que mucho o bastante (Gráfico 11). Por otra parte, el $77 \%$ valora que los niños violentos proceden de familias desestructuradas y, el $93 \%$ de barrios marginales (Gráfico 12).

Actualmente, se está realizando una investigación más amplia sobre la violencia contra el profesorado y su incidencia en Educación Primaria y Secundaria. Como puede comprobarse en el Gráfico 10, la mayoría del alumnado universitario que ha participa- 
do en la investigación valora que, en la Universidad, existe muy poca violencia contra el profesorado.

Resultan de gran interés las conclusiones relativas a violencia escolar y alumnado inmigrante, resaltando el 64\% que sufren más acoso, mientras que sólo el 17\% valora que son más violentos. Sin embargo, el 92\% considera que los problemas de la inmigración influyen en el aumento de violencia (Gráfico 13).

La mayoría del alumnado universitario muestra preocupación por el aumento de la violencia en la sociedad actual (72\%); sin embargo, no indica posibles actuaciones para evitarla (Gráfico 15). Por otra parte, sólo el 15\% reconoce que no suelen enviar -o recibir- correos electrónicos amenazantes (Gráfico 16) o utilizar el móvil para enviar -o recibir- mensajes, todos, etc., de acoso a otros/as compañeros/as (Gráfico 17).

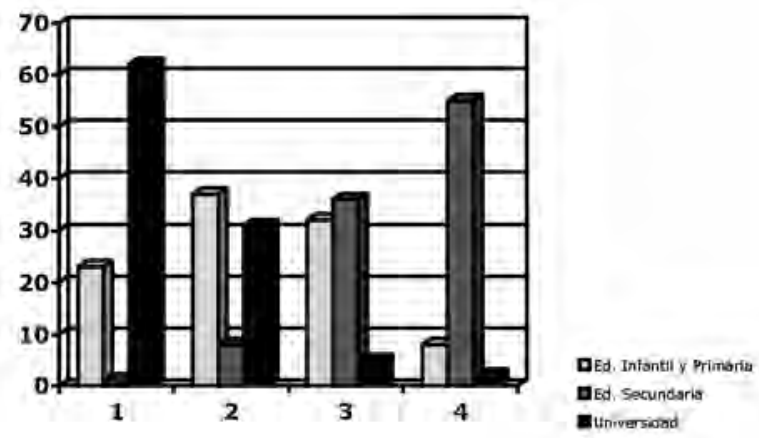

Gráfico 1. Violencia en los centros

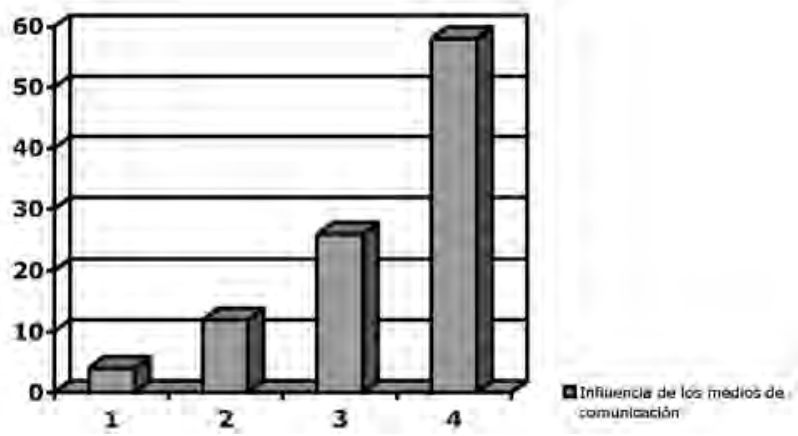

Gráfico 2. Medios de comunicacón y violencia 


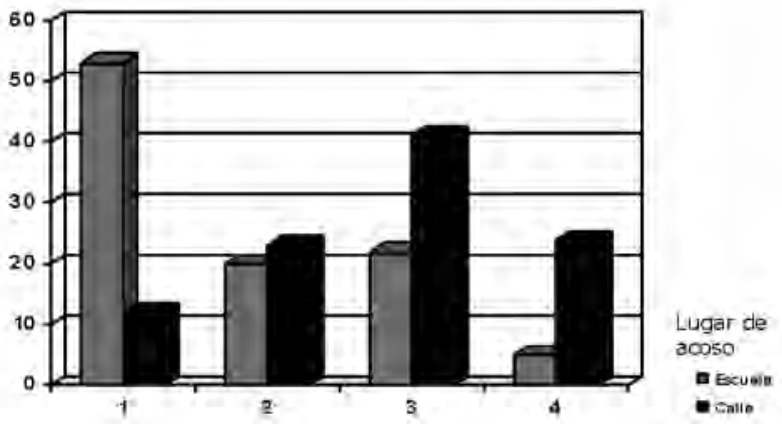

Gráfico 3. Lugar de acoso

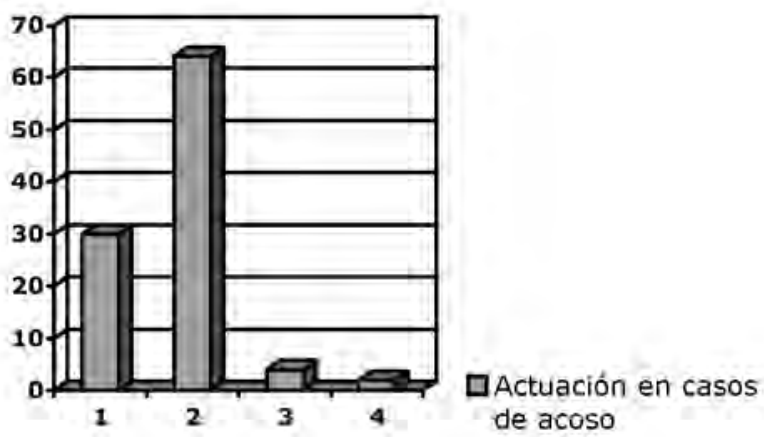

Gráfico 4. Actuación en casos de acoso

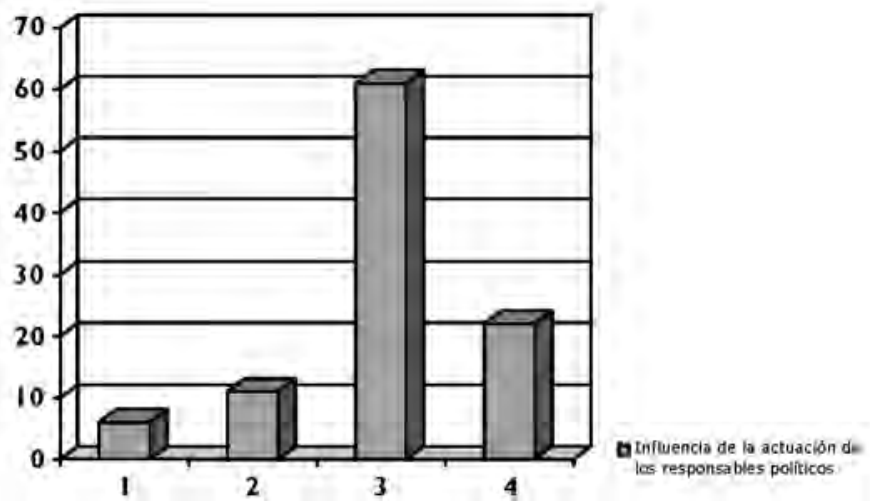

Gráfico 5. Comportamiento de los responsables políticos y violencia 


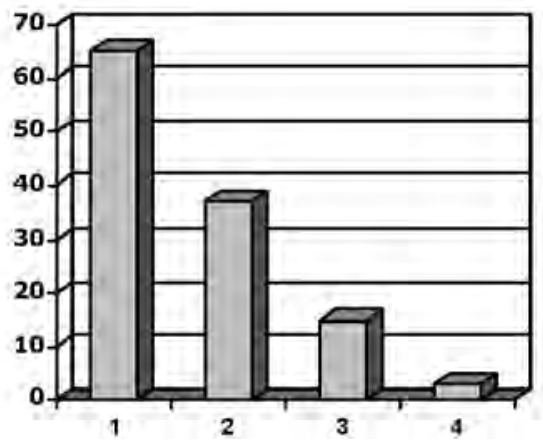

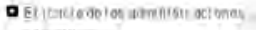
nomatry

Gráfico 6. Eficacia de las administraciones educativas

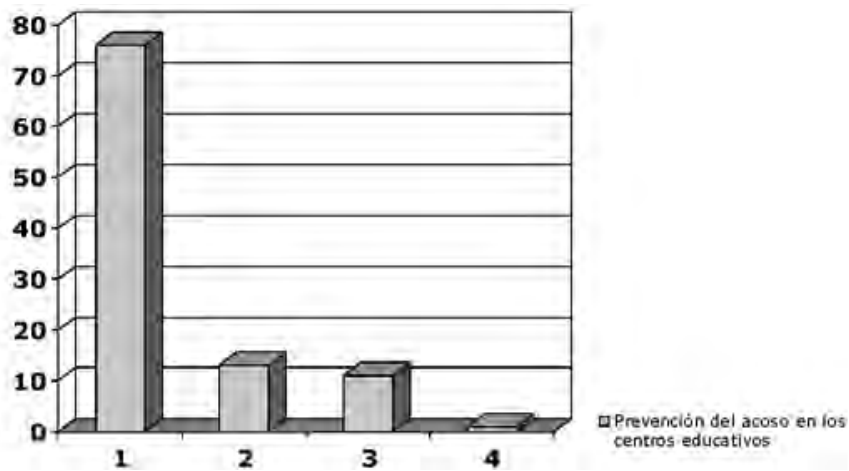

Gráfico 7. Prevención del acoso en los centros

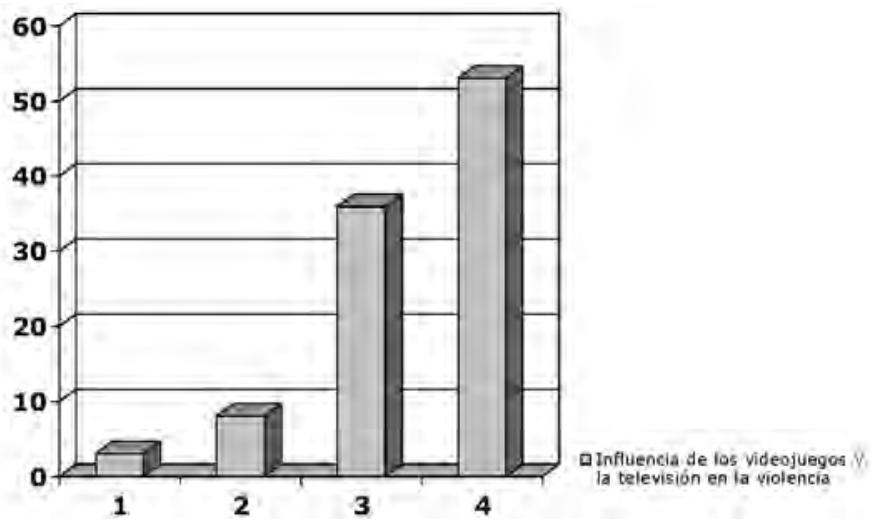

Gráfico 8. Influencia de los videojuevos y la tv en la violencia 


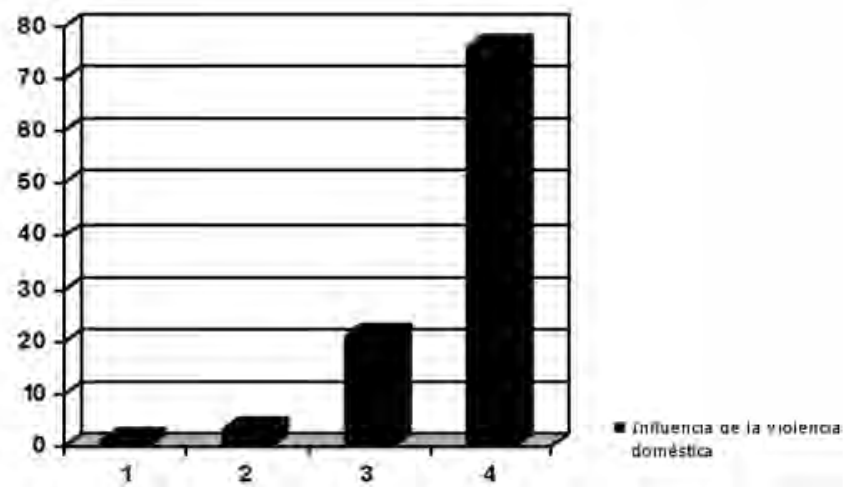

Gráfico 9. Violencia doméstica y acoso en las aulas

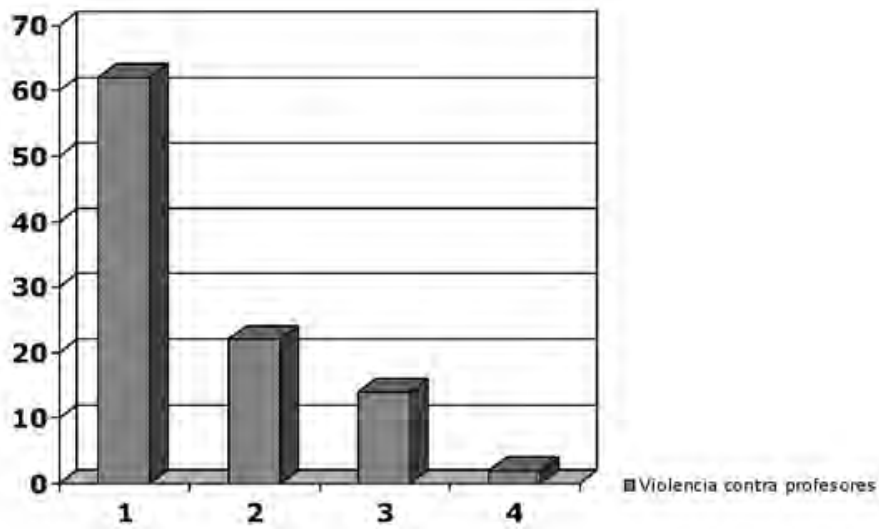

Gráfico 10. Violencia contra profesores

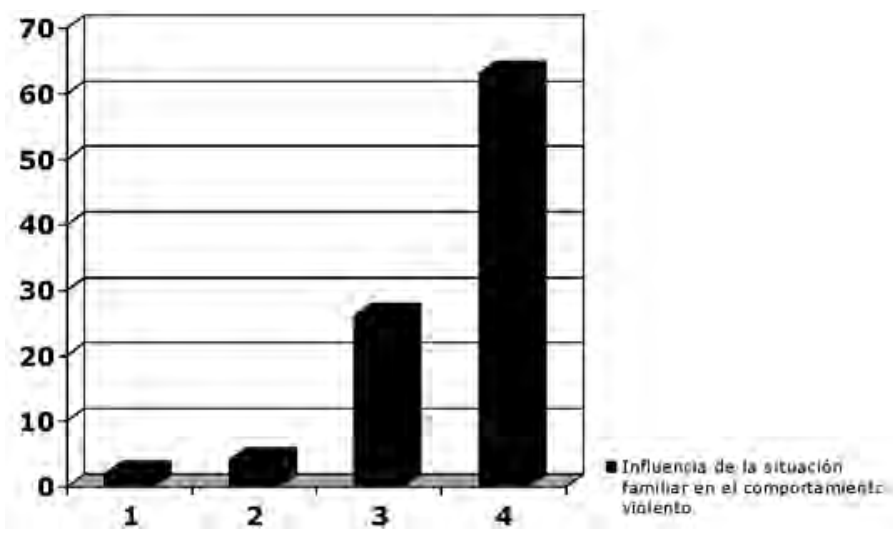

Gráfico 11. Influencia de la situación familiar en el comportamiento violento 


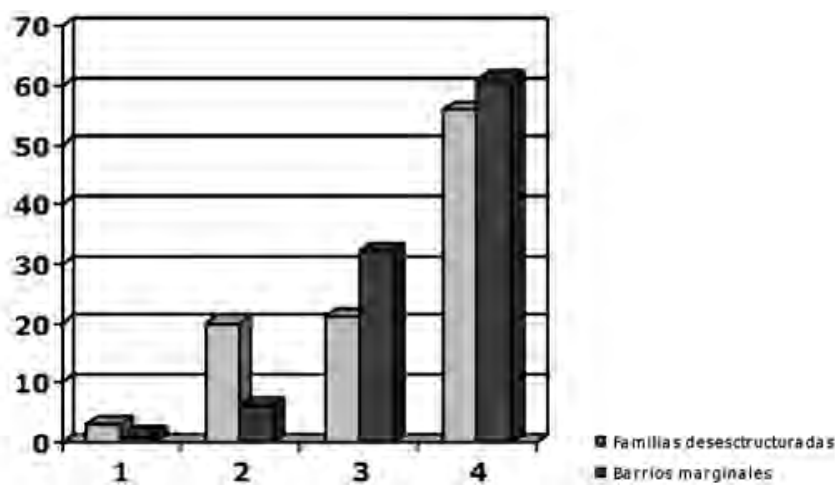

Gráfico 12. Procedencia social de los niños violentos

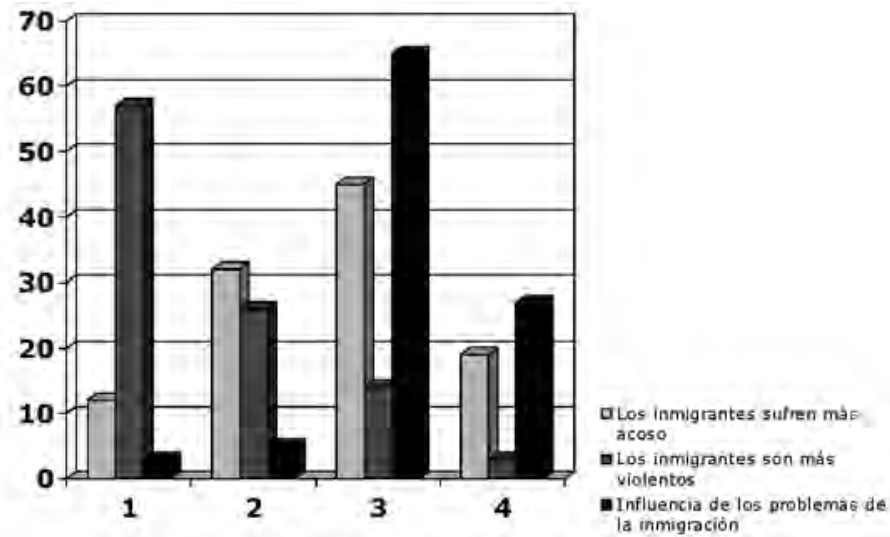

Gráfico 13. Violencia escolar y alumnado inmigrante

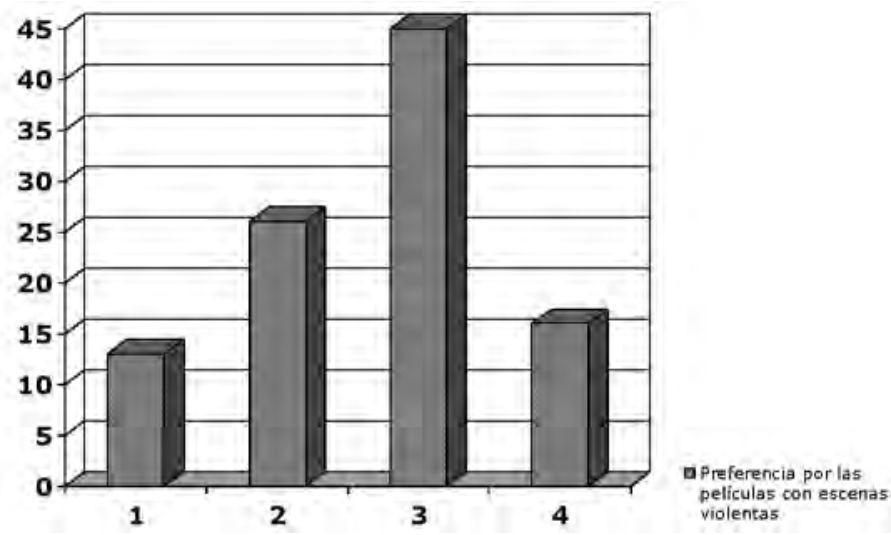

Gráfico 14. Preferencia por las películas con escenas violentas 


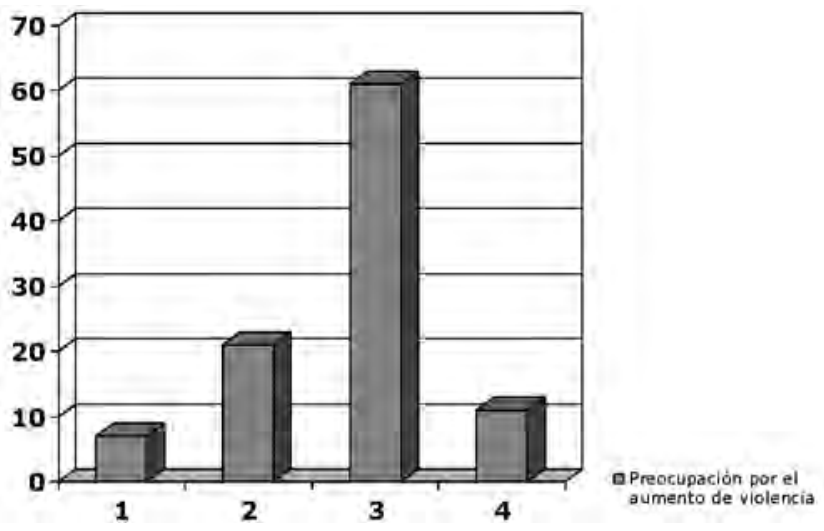

Gráfico 15. Preocupación por el aumento de violencia

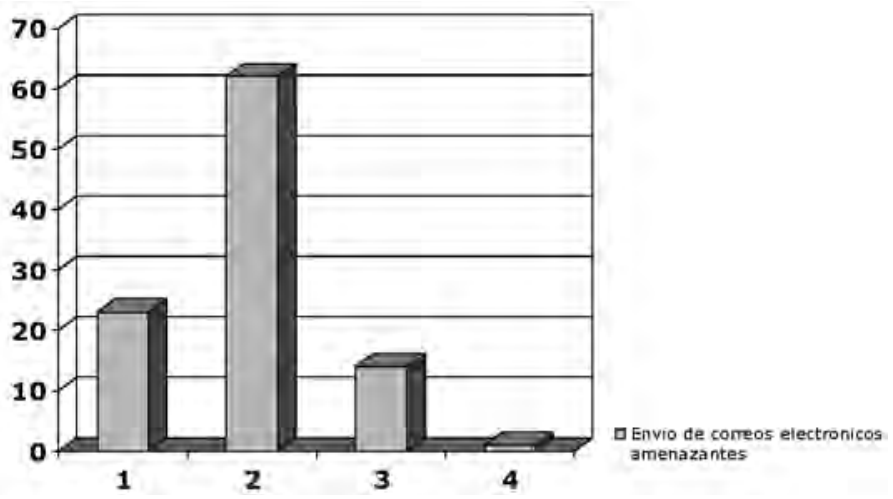

Gráfico 16. Correos electrónicos amenazantes

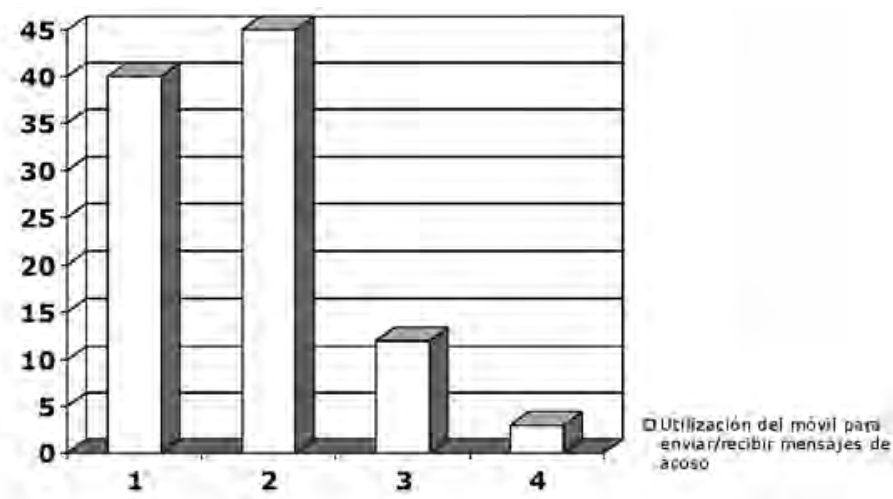

Gráfico 17. Utilización del móvil para acosar a otros compañeros 


\section{Conclusiones más significativas}

Ha quedado claramente demostrada la ineficacia de la Administración educativa en este problema, por lo que es preciso exigirle responsabilidades políticas y sociales, para que supere el falso debate de las cifras, olvide el síndrome de la negación institucional y haga frente - de una vez por todas- al problema de la violencia en las aulas.

Las pautas imperantes en nuestro sistema educativo mantienen las condiciones de desigualdad del alumnado, reforzadas culturalmente por los medios de comunicación y los fenómenos de masas, que suelen resultar alienantes para el ser humano, enajenándole de las posibilidad de unos planteamientos propios y subsumiéndole en un modelo cultural -o subcultural- ajeno a lo que debería constituir la preocupación de toda persona.

Por otra aparte, el profesorado suele reproducir -en sus actividades- el sistema de poder, estableciendo un evidente mecanismo de refuerzo y configurando la legitimación de una estructura social inadecuada, incluso por encima de los aspectos didácticos que conlleva la transmisión y elaboración de conocimientos. A mayor abundamiento, se está produciendo una fuerte contradicción entre los principios pedagógicos que -teóricamente- deben inspirar la práctica docente y esa misma práctica.

La interiorización de las pautas de conducta imperantes en nuestra sociedad y la autorrepresión sistemática que ellas representan son posiblemente los apartados ideológicos más ocultos y eficaces del Estado, al ser refrendados tácitamente por la familia del alumnado. De mantenerse esta situación, el sistema de "domesticación industrial" de seres humanos sumisos deja escasas esperanzas para que nuestra futura ciudadanía sea feliz, solidaria y auténticamente responsable.

Existe una evidente contradicción entre los objetivos teóricos del sistema escolar y la realidad. La práctica de una escuela transformadora encuentra fuertes barreras que la convierten en inviable, no permitiéndose que la educación sea motor de cambio y generándose cierto desencanto entre el personal docente.

El entorno debe ser generador de riqueza cultural para la persona, como punto de partida; pero nunca reducir al mismo los objetivos planteados. Hay que intentar llegar más allá, conociendo las múltiples realidades que otros entornos nos ofrecen. Nunca hay que olvidar que la cultura del trabajo -restringida al entorno- puede provocar una limitación de las posibilidades del desarrollo personal.

Resulta ineludible el acercamiento de la Escuela a su entorno, comenzando por los padres y madres del alumnado.

Si consideramos el insuficiente avance real que -generalmente- se está produciendo en la gestión democrática de los Centros y en la participación de los distintos sectores de la comunidad escolar, no hay que ser demasiado optimistas respecto a un adecuado tratamiento de este problema.

El profesorado debe asumir que las características del alumnado -positivas o negativas- están configuradas, como siempre, por la época que les ha tocado vivir, resultando evidente que es capaz de autogobernarse en gran medida, de plantear ideas sobre su propia educación y de participar con entusiasmo en tareas y proyectos ilusionadores (incluso a costa de su tiempo libre), formando grupos de trabajo ajenos al estricto currículo 
escolar. Por otra parte, debe considerase que el primer derecho del alumnado es tener una Escuela de calidad, garantizada por los poderes públicos y en la que no existan discriminaciones de ningún tipo. Dicha Escuela debe ser pública, plural, creativa, compensadora de desigualdades, crítica e investigadora, así como propiciar posibilidades reales de desarrollo social, personal y profesional para todos los alumnos y alumnas, como derecho básico e inalienable.

Una vez más, resulta necesario insistir en que nos encontramos ante un problema social que requiere un planteamiento holístico, y que los primeros en cambiar actitudes deben ser nuestros representantes públicos, sindicatos incluidos. No debemos olvidar que la mayoría de los alumnos que muestran conductas agresivas en la infancia suelen convertirse en adultos violentos. Por tanto, la educación para la paz, la no violencia, la tolerancia y la solidaridad, desde la más temprana edad, constituyen el mejor medio para prevenir estas situaciones y la también preocupante violencia doméstica. Una tarea en la que deben trabajar colaborativamente, no sólo los padres y docentes, sino la sociedad, en general.

\section{Anexo I}

\section{CUESTIONARIO SOBRE VIOLENCIA EN LOS CENTROS ESCOLARES}

\section{Introducción}

Diariamente, se difunden informaciones sobre nuevas víctimas de la violencia en los Centros escolares, tanto públicos como privados, generando una alarma social que precisa un amplio análisis -con rigor científico y metodológico- de los datos ofrecidos.

Por ello, le pedimos que conteste a la Encuesta de la forma más sincera posible, ya que no le compromete a nada, mientras que, para nosotros, es muy importante su opinión. No existen buenas ni malas respuestas; lo efectivo es que indique su opinión personal.

La mayoría de las cuestiones planteadas proponen una serie de alternativas, entre las que puede elegir la que esté más de acuerdo con su opinión personal. Basta con que ponga una cruz en el cuadro correspondiente. En algún caso, le ofrecemos un pequeño espacio para expresarse. En otros, se le ofrece una escala con valoraciones de 1, 2, 3, 4, (1 es el valor mínimo y 4 , el máximo. Es decir, 1 = nada; 2 = poco; $3=$ bastante; $4=$ mucho). Además, si quiere manifestar cualquier otra opinión, puede hacerlo con libertad.

\section{Gracias por su valiosa colaboración}




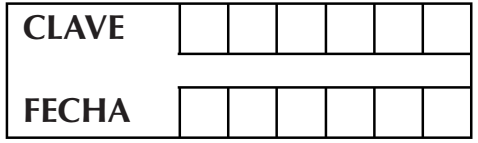

\section{A) DATOS DE LA PERSONA ENTREVISTADA}

1. Sexo: Varón $\square \quad$ Mujer $\square$

2. Edad:__ años 3. Lugar de nacimiento:

4. Situación profesional:
4.1. Estudia:
CARRERA:

4.2. Trabaja: Situación laboral:

4.3. Paro: $\square$

5. Titulación académica:

5.1. Diplomado:

5.2. Licenciado:

5.3. Otras (especificar):

6. Centro de trabajo:

7. Años de experiencia:

8. Otros datos de interés: 


\section{A) Evaluación de la violencia y bullying}

\begin{tabular}{|c|c|c|c|c|c|}
\hline \multicolumn{2}{|c|}{ Indicadores } & \multirow[t]{2}{*}{1} & \multirow[t]{2}{*}{2} & \multirow[t]{2}{*}{3} & \multirow[t]{2}{*}{4} \\
\hline 1 & $\begin{array}{l}\text { Presencia de lesiones en el rostro, cuello, espalda, nalgas, } \\
\text { muslos }\end{array}$ & & & & \\
\hline 2 & Moratones con formas extrañas & & & & \\
\hline 3 & $\begin{array}{l}\text { Quemaduras extrañas (producidas por cigarrillos, } \\
\text { puros, etc.) }\end{array}$ & & & & \\
\hline 4 & Señales de mordisco humano & & & & \\
\hline 5 & Derrames oculares & & & & \\
\hline 6 & Falta de mechones de cabello & & & & \\
\hline 7 & Tiene dificultades para sentarse o caminar & & & & \\
\hline 8 & Llora a veces sin motivo aparente & & & & \\
\hline 9 & $\begin{array}{l}\text { Explicaciones poco convincentes o extrañas respecto } \\
\text { a la lesión }\end{array}$ & & & & \\
\hline 10 & Es excesivamente responsable en su trabajo escolar & & & & \\
\hline 11 & Está, siempre que es posible, con el profesor/a & & & & \\
\hline 12 & Asiste al Colegio sin desayunar, comer, etc. & & & & \\
\hline 13 & Necesidades médicas básicas sin atender & & & & \\
\hline 14 & Normalmente no va aseado al Colegio & & & & \\
\hline 15 & Se duerme en clase y parece cansado/a & & & & \\
\hline 16 & Demuestra miedo a sus padres o cuidadores & & & & \\
\hline 17 & $\begin{array}{l}\text { No quiere ir a su casa y pasa muchas horas en la calle o } \\
\text { en casa de otros amigos }\end{array}$ & & & & \\
\hline 18 & Rehuye de hablar de su familia & & & & \\
\hline 19 & $\begin{array}{l}\text { Ejerce funciones parentales con sus hermanos } \\
\text { más pequeños }\end{array}$ & & & & \\
\hline 20 & Se relaciona con otros niños mayores físicamente & & & & \\
\hline 21 & $\begin{array}{l}\text { Se muestra agresivo con sus compañeros y con personas } \\
\text { adultas }\end{array}$ & & & & \\
\hline 22 & $\begin{array}{l}\text { Suele llevar regalos, comida, dinero... para otros } \\
\text { compañeros }\end{array}$ & & & & \\
\hline 23 & Tendencia a la soledad, tristeza, mutismo & & & & \\
\hline 24 & Presenta conductas violentas y a veces se autolesiona & & & & \\
\hline 25 & Se refugia en los videojuegos y en la TV & & & & \\
\hline 26 & Intenta llamar la atención constantemente & & & & \\
\hline 27 & $\begin{array}{l}\text { Utiliza las amenazas y la violencia para conseguir } \\
\text { sus intereses }\end{array}$ & & & & \\
\hline 28 & Es tirano y prepotente con sus iguales & & & & \\
\hline
\end{tabular}




\section{A) Evaluación de la violencia y bullying (continuación)}

\begin{tabular}{|c|c|c|c|c|c|}
\hline \multicolumn{2}{|c|}{ Indicadores } & \multirow[t]{2}{*}{1} & \multirow[t]{2}{*}{2} & \multirow[t]{2}{*}{3} & \multirow[t]{2}{*}{4} \\
\hline 29 & Miente con frecuencia & & & & \\
\hline 30 & Es líder en conductas asociales & & & & \\
\hline 31 & Cambia constantemente de humor & & & & \\
\hline 32 & Baja autoestima & & & & \\
\hline 33 & Gran dificultad en la resolución de conflictos & & & & \\
\hline 34 & Desconfiado de los adultos y de sus compañeros & & & & \\
\hline 35 & Presenta conductas asociales: hurtos, fugas, vandalismo,... & & & & \\
\hline 36 & Ha tenido intentos de suicidio & & & & \\
\hline 37 & No colabora en las actividades de grupo & & & & \\
\hline 38 & Muestra total apatía ante cualquier actividad & & & & \\
\hline 39 & Absentismo escolar & & & & \\
\hline 40 & Tiene grandes dificultades para prestar atención & & & & \\
\hline 41 & $\begin{array}{l}\text { Suele boicotear las actividades de clase y busca llamar } \\
\text { al atención }\end{array}$ & & & & \\
\hline 42 & No realiza los trabajos individuales ni los deberes & & & & \\
\hline 43 & Se cansa con facilidad & & & & \\
\hline 44 & Presenta dificultades en la comunicación oral & & & & \\
\hline 45 & Descuida el material escolar & & & & \\
\hline 46 & Tiene problemas de aprendizaje & & & & \\
\hline 47 & $\begin{array}{l}\text { Siente angustia y rechazo cuando tiene que cambiarse } \\
\text { de ropa delante de otras personas }\end{array}$ & & & & \\
\hline 48 & $\begin{array}{l}\text { Dispone de conocimientos y/o conductas sexuales } \\
\text { inadecuadas para su edad }\end{array}$ & & & & \\
\hline 49 & Utiliza un vocabulario inapropiado para su edad & & & & \\
\hline 50 & $\begin{array}{l}\text { Dispone de material pornográfico u otros materiales } \\
\text { sexuales }\end{array}$ & & & & \\
\hline 51 & $\begin{array}{l}\text { Pasa muchas horas con el ordenador y recibe/envía } \\
\text { correos electrónicos amenazantes }\end{array}$ & & & & \\
\hline 52 & $\begin{array}{l}\text { Utiliza el móvil para recibir o enviar mensajes, fotos, etc. } \\
\text { de acoso a otros compañeros }\end{array}$ & & & & \\
\hline
\end{tabular}




\section{B) Violencia y educación en la sociedad actual}

\begin{tabular}{|c|c|c|c|c|c|}
\hline \multicolumn{2}{|c|}{ Dificultades } & \multirow[t]{2}{*}{1} & \multirow[t]{2}{*}{2} & \multirow[t]{2}{*}{3} & \multirow[t]{2}{*}{4} \\
\hline 1 & Conozco lo que significa "bullyng" & & & & \\
\hline 2 & $\begin{array}{l}\text { Considero que, en la actualidad, hay violencia en los } \\
\text { Centros de E. Infantil y E. Primaria }\end{array}$ & & & & \\
\hline 3 & $\begin{array}{l}\text { Estimo que, en la actualidad, hay violencia en los } \\
\text { Centros de E. Secundaria }\end{array}$ & & & & \\
\hline 4 & $\begin{array}{l}\text { Considero que, en la actualidad, hay violencia en } \\
\text { la Universidad }\end{array}$ & & & & \\
\hline 5 & $\begin{array}{l}\text { Los medios de comunicación están influyendo en el } \\
\text { aumento de violencia }\end{array}$ & & & & \\
\hline 6 & Personalmente, me he sentido acosado/a en la Escuela & & & & \\
\hline 7 & Personalmente, me he sentido acosado/a en la calle & & & & \\
\hline 8 & Cuando he detectado algún caso de acoso, he actuado & & & & \\
\hline 9 & $\begin{array}{l}\text { El comportamiento de los responsables políticos } \\
\text { influye en el aumento de violencia }\end{array}$ & & & & \\
\hline 10 & $\begin{array}{l}\text { Las Administraciones educativas actúan con eficacia } \\
\text { en los casos denunciados }\end{array}$ & & & & \\
\hline 11 & $\begin{array}{l}\text { En los Centros educativos, se detecta rápidamente a la } \\
\text { victima y la sanción es también inmediata. }\end{array}$ & & & & \\
\hline 12 & $\begin{array}{l}\text { Se realiza un auténtico proceso de prevención del acoso } \\
\text { en los Centros educativos }\end{array}$ & & & & \\
\hline 13 & $\begin{array}{l}\text { El acoso psicológico es mayor en las niñas } \\
\text { que en los niños }\end{array}$ & & & & \\
\hline 14 & $\begin{array}{l}\text { Los videojuegos y la TV influyen en el aumento } \\
\text { de violencia }\end{array}$ & & & & \\
\hline 15 & $\begin{array}{l}\text { La violencia doméstica tiene relación directa con el } \\
\text { acoso en las aulas }\end{array}$ & & & & \\
\hline 16 & $\begin{array}{l}\text { Los/as niños/as más débiles (física o mentalmente) } \\
\text { sufren más el acoso }\end{array}$ & & & & \\
\hline 17 & Se tiene miedo a denunciar a los acosadores & & & & \\
\hline 18 & El patio de recreo es donde más se produce el acoso & & & & \\
\hline 19 & $\begin{array}{l}\text { Los profesores no suelen preocuparse en tomar medidas } \\
\text { efectivas contra los acosadores }\end{array}$ & & & & \\
\hline 20 & He vivido situaciones de violencia contra profesores & & & & \\
\hline 21 & $\begin{array}{l}\text { La situación familiar influye en el comportamiento } \\
\text { violento }\end{array}$ & & & & \\
\hline 22 & $\begin{array}{l}\text { Los/as niños/as violentos/as suelen proceder } \\
\text { de familias desestructuradas }\end{array}$ & & & & \\
\hline
\end{tabular}




\section{B) Violencia y educación en la sociedad actual (continuación)}

\begin{tabular}{|c|c|c|c|c|c|}
\hline \multicolumn{2}{|c|}{ Dificultades } & \multirow[t]{2}{*}{1} & \multirow[t]{2}{*}{2} & \multirow[t]{2}{*}{3} & \multirow[t]{2}{*}{4} \\
\hline 23 & $\begin{array}{l}\text { Los/as niños/as violentos/as suelen proceder de barrios } \\
\text { marginales }\end{array}$ & & & & \\
\hline 24 & Los inmigrantes sufren más acoso escolar & & & & \\
\hline 25 & Los inmigrantes son más violentos & & & & \\
\hline 26 & $\begin{array}{l}\text { Los problemas de la inmigración influyen en el aumento } \\
\text { de la violencia }\end{array}$ & & & & \\
\hline 27 & $\begin{array}{l}\text { Las víctimas de la violencia son sujetos inseguros que } \\
\text { sufren en silencio las agresiones }\end{array}$ & & & & \\
\hline 28 & $\begin{array}{l}\text { Los/as niños niños/as que viven en un ambiente de } \\
\text { violencia familiar se convierten en agresores }\end{array}$ & & & & \\
\hline 29 & Me gustan las películas con escenas violentas & & & & \\
\hline 30 & $\begin{array}{l}\text { Personalmente, me preocupa el aumento de violencia } \\
\text { en la sociedad actual }\end{array}$ & & & & \\
\hline 31 & $\begin{array}{l}\text { Paso muchas horas con el ordenador y recibo y/o envío } \\
\text { correos electrónicos amenazantes }\end{array}$ & & & & \\
\hline 32 & $\begin{array}{l}\text { Utilizo el móvil para recibir o enviar mensajes, fotos, etc. } \\
\text { de acoso a otros compañeros }\end{array}$ & & & & \\
\hline
\end{tabular}

\section{Referencias bibliográficas}

Beck, U. (2004). Poder y contra-poder en la era global. Barcelona: Paidós.

Boqué, M .C. y otros (2005). Hagamos las paces. Mediación 3-6 años. Barcelona: CEAC.

Burnley, J. (1993). Conflicto. Madrid: Morata.

Campbell, D.T. y Stanley, J.C. (1978). Diseños experimentales y cuasiexperimentales en la investigación social. Buenos Aires: Amorrortu.

Cerezo, F. (Coord) (1998). Conductas agresivas en la edad escolar. Madrid: Pirámide.

COLECTIVO AMANI (2004). La escuela intercultural: regulación de conflictos en contextos multiculturales. Madrid: Catarata.

COMISIÓN EUROPEA (2002). Educación y formación en Europa: sistemas diversos, objetivos compartidos para 2010. Luxemburgo: Oficina de Publicaciones Oficiales de las Comunidades Europeas.

CONSEJO ESCOLAR DE CLM (2007). El día a día en los Centros escolares (Jornada de reflexión y debate, 27 Abril 2006). Toledo: Consejo Escolar de CLM.

Curwin, R.L. y Mendler, A.N. (1987). La disciplina en clase. Organización del centro y del aula. Madrid: Narcea.

Debarbieux, E. (1997). "La violencia en la escuela francesa: Análisis de la situación, políticas públicas e investigaciones". Revista de Educación, 313, pp. 79-94 
DEFENSOR DE LA COMUNIDAD DE MADRID (1999). Convivir es vivir. Delegación del Gobierno de Madrid.

Díaz Aguado, M.J. (2006). Del acoso escolar a la cooperación en las aulas. Madrid: Pearson.

Dominguez, T. y otros (1996). Comportamientos no violentos. Propuestas interdisciplinares para construir la paz. Madrid: Narcea.

Erezo, F. (Coord.) (1998). Conductas agresivas en la edad escolar. Madrid: Ed. Pirámide.

Fernández García, I. (2002). Guía para la convivencia en el aula. Barcelona: Praxis.

Funk, W. (1997). "Violencia escolar en Alemania". Revista de Educación, 313, pp. 5378.

Garcia Garrido, J. L. (Ed.) (2008). Formar ciudadanos europeos. Madrid: Academia de Ciencias y Artes.

Gimeno Sacristán, J. (2002). "Dilemas y opciones". En Sacristán, A. Lecturas de Didáctica. Madrid: Cuadernos de la UNED (pp. 15-28).

Guba. E.G. (1989). "Criterios de credibilidad en la investigación naturalista". En Gimeno, J. y Pérez Gómez, A.I. (Eds.). La enseñanza: su teoría y su práctica. Madrid: Akal, (pp.148-165).

Imbernon, F. (2002). La investigación educativa como herramienta de formación del profesorado. Barcelona: Graó.

Jares, X.R. (2006). Pedagogía de la convivencia. Barcelona: Graó.

Marina, J. A. (2002). "Profesores para un mundo ultramoderno". Cuadernos de Pedagogía, no 304 .

Martínez Bonafé, J. (2002). Políticas del libro de texto escolar. Madrid: Morata.

Mayor Zaragoza, F. (2000). Un Món nou. Barcelona: Centro UNESCO de Cataluña.

Melero Martín, J. (1993). Conflictividad y violencia en los centros. Madrid: Siglo XXI.

Muñoz Cantos, J. (2004). "Sociedad y educación, aquí y ahora". La Verdad, 9 abril.

Ohsako, T. (1998). Violence at school. Global sigues and interventions. UNESCO.

Ortega, R. (2003). La violencia escolar. Estrategias de intervención. Barcelona: Graó.

Palomares, A. (2004). Profesorado y educación para la diversidad en el siglo XXI. Cuenca: Universidad de Castilla-La Mancha.

Palomares, A. (2005). "El acoso escolar: una asignatura pendiente". En la Tribuna de Albacete, 4-11, pág. 4.

Palomares, A. (2006). "El acoso escolar, un grave problema social". En http://firgoa.usc.es/drupal/node/31951.

Palomares, A. (2006). "La nueva Ley educativa y los intereses políticos". (http://firgoa.usc.es/drupal/node, 2-05-06).

Palomares, A. (2007). "La violencia en los Centros Escolares". Ponencia presentada en el Congreso Mundial de Educación de la Infancia para la Paz. Albacete.

Palomares, A. (2007). Nuevos retos educativos. El modelo docente en el Espacio Europeo. Cuenca: Servicio de Publicaciones de la Universidad de Castilla-La Mancha. 
Pérez Gómez, A.I. (2003). Más allá del academicismo. Los desafíos de la Escuela en la era de la información y la perplejidad. Málaga: Servicio de Publicaciones de la Universidad.

Sahuquillo, M.R. (2008). "El acoso escolar desciende después del caso Jokin". El País, 20-Febrero, pág. 50.

Santos Guerra, M.A. (coord.) (2003). Aprender a convivir en la escuela. Madrid: Akal.

Sastre, G. y Moreno, M. (2005). Resolución de conflictos y aprendizaje emocional. Una perspectiva de género. Barcelona: CEAC.

Segura Morales, M. (2006). Enseñar a convivir no es tan difícil. Para quienes no saben que hacer con sus alumnos. Bilbao: Desclée de Brourwer.

Torrego, J.C. y Moreno, J.M. (2003). Convivencia y Disciplina en la Escuela. Madrid: Alianza Editorial.

Tuvilla Rayo, J. (Comp.) (1994). La escuela: Instrumento de Paz y Solidaridad. Sevilla: Ed. MECEP.

UNESCO (1998). La educación Superior en el Siglo XXI: Visión y Acción. Conferencia Mundial sobre educación Superior. París, 5-9 de octubre.

UNESCO (1999). Declaration on Science and the Science Agenda: Framework Action. París: UNESCO.

Vaello, J. (2003). Resolución de conflictos en el aula. Madrid: Santillana.

Wihtol de Wenden, C. (1999). La ciudadanía europea. Barcelona: Bellaterra. 